\title{
Transformações de dados em experimentos com abobrinha italiana em ambiente protegido
}

\author{
Data transformations in experiments with zucchini in protected environment
}

\author{
Mara Rubia Machado Couto ${ }^{I}$ Alessandro Dal'Col Lúcio ${ }^{I I *}$ Sidinei José Lopes ${ }^{I I}$ \\ Ricardo Howes Carpes ${ }^{\text {III }}$
}

RESUMO

Em olericultura, o interesse é a produção de frutos comercializáveis. Nesses experimentos, comumente são avaliadas variáveis, tais como a fitomassa e o número de frutos. No entanto, essas variáveis apresentam uma característica particular que está relacionada com sua ausência ou presença no momento da colheita. Esse atributo é representado por valores zeros, para essas variáveis, gerando certa variabilidade e afetando a análise de variância dos dados. Este trabalho teve por objetivo definir uma transformação adequada para as variáveis observadas em experimentos com a cultura da abobrinha italiana, realizados em ambiente protegido, visando a estabilizar a variabilidade gerada pela presença de valores zeros nas múltiplas colheitas dos frutos. Foram analisados experimentos com a cultura da abobrinha italiana utilizando a família de transformações de Box-Cox, com uma adaptação apresentada em YAMAMURA (1999). Concluiu-se que, para experimentos com abobrinha italiana, avaliando a fitomassa e o número de frutos, a transformação indicada é a raiz quarta. Mesmo com redução na variabilidade e a normalidade dos resíduos, observadas em todos os experimentos, o uso do método não foi eficiente para tornar as variâncias homocedásticas.

Palavras-chave: Box-Cox, análise de variância, coeficiente de variação, variância heterocedástica.

\section{ABSTRACT}

The interest in horticulture is the production of marketable fruit. In such experiments, phytomass and the number of fruits are commonly measured variables. However, these variables have a particular characteristic related to their absence or presence at harvest time. This attribute is represented by zero values for these variables, generating some variability and affecting the variance analysis. This work aimed to define an appropriate data transformation for the variables observed in experiments with the zucchini culture, realized in protected environment, designed to stabilize the variability values generated by the presence of zeros in harvest multiple fruit. The analysis was based on experiments with the zucchini culture , using the family of the Box-Cox transformation, with an adaptation made by YAMAMURA (1999). It was concluded that for experiments with zucchini, assessing the phytomass and number of fruits, the square square root transformation is indicated. The same was observed with the reduction in variability and normal errors, observed in all experiments. The use of the method was not efficient to make the variances homocedastic.

Key words: Box-Cox, variance analysis, coefficient variation, heterocedastic variances.

\section{INTRODUÇÃO}

A abobrinha italiana (Curcubita pepo), planta da família das cucurbitáceas, é uma das 10 hortaliças de maior valor econômico e de maior produção no Brasil, principalmente nas regiões Centro e Sul do país. Tem ciclo de 50 a 80 dias, podendo ser cultivada em campo, tanto no verão, quanto na primavera (CAMARGO, 1981). Em cultivo protegido, além desses períodos, pode ser produzida no outono e inverno, quando o preço por quilo pode triplicar. Com isso, no Estado do Rio Grande do Sul, o cultivo da abobrinha italiana em estufas plásticas é uma

\footnotetext{
IDepartamento de Estatística, Centro de Ciências Naturais e Exatas (CCNE), Universidade Federal de Santa Maria (UFSM), Santa Maria, RS, Brasil.

IIDepartamento de Fitotecnia, Centro de Ciências Rurais (CCR), UFSM, 97105-900, Santa Maria, RS, Brasil. E-mail: adlucio@smail.ufsm.br.*Autor para correspondência.

IIIPrograma de Pós-graduação em Agronomia, UFSM, Santa Maria, RS, Brasil.
} 
alternativa para os produtores aumentarem seus rendimentos líquidos, devido à redução de perdas e ao aumento da produtividade. De acordo com STRECK et al. (2003), a produtividade pode alcançar mais que o dobro da relatada por FILGUEIRA (2000), que cita um rendimento de cerca de oito toneladas para cultivos em campo, além de apresentar vantagens, principalmente na qualidade superior dos produtos e produção em baixas temperaturas do ar.

Em cultivos olericolas sob ambiente protegido, a maioria dos tratos culturais são realizados de forma individualizada por planta e o mais uniforme possível. Porém, alguns autores relatam a existência de variabilidade da produção de frutos entre as linhas de cultivo, o que pode prejudicar a condução de experimentos nessas condições. SOUZA et al. (2002) relataram grande variabilidade para crescimento dos frutos de abobrinha italiana no decorrer do ciclo produtivo e, da mesma forma, LÚCIO et al. (2003) verificaram que todas as colheitas de pimentão apresentaram heterogeneidade entre as linhas de cultivo. Já LORENTZ et al. (2005), trabalhando com pepineiro, verificaram que, em $46 \%$ das colheitas, as linhas de cultivo se mostraram com variâncias heterogêneas, observando também grandes oscilações das médias da produção entre as linhas de cultivo. Também LOPES et al. (1998) relataram mudanças na concentração da produção de tomate no decorrer das colheitas e constataram diferenças de produção entre as linhas de cultivo.

A variabilidade da produção entre plantas sob cultivo protegido pode acarretar heterogeneidade de variâncias, que deverá ser evitada para a realização da análise de variância e, assim, assegurar o nível de significância dos testes $\mathrm{F}$ e das análises complementares. Em análises de dados experimentais com variâncias heterogêneas, o nível de significância passa a ficar acima do especificado (CONAGIN et al., 1993). Assim, tanto o estudo do comportamento da média, quanto o da variância da produção em ambiente protegido, ao longo de diferentes colheitas, se faz necessário. Além disso, a identificação da interferência e da relação dos diferentes manejos culturais naquelas estatísticas também são importantes, pois são fatores que podem afetar os resultados obtidos em trabalhos científicos.

No cultivo de olericolas, a produção de frutos comercializáveis é de grande interesse. No entanto, a ocorrência de valores zeros é bastante frequente, pois nem sempre existem frutos a serem colhidos ou nem sempre estes possuem tamanho adequado para sua colheita e comercialização (SOUZA et al., 2002; LORENTZ et al., 2005; LÚCIO et al., 2008).
Essa frequência de zeros gera variabilidade, o que pode provocar distorções nos procedimentos de inferência derivados da análise de variância. Nesses casos, o uso de transformações de variáveis pode ser uma alternativa para atender à pressuposição da homogeneidade das variâncias residuais na análise de variância. Em experimentos em que há variáveis que apresentam como resultados o valor zero, como é o caso de experimentos com culturas olericolas com múltiplas colheitas nas quais nem todas as plantas apresentam frutos aptos a serem colhidos em determinada colheita, a eficácia das transformações fica prejudicada devido à presença de superdispersão que, segundo BORGATTO et al. (2006), pode ocorrer devido à variabilidade da média e ao excesso de zero.

Ao se dispor de um conjunto de dados com vários valores iguais a zero, é comum o acréscimo de uma constante (c) a cada um dos dados (STORCK et al., 2000; BANZATTO \& KRONKA, 2006). A determinação do valor constante $c$ que deve ser adicionada à variável original $(\mathrm{Y})$ tem sido bastante discutida na literatura, mas, geralmente, assume-se $c=$ 1. Conforme YAMAMURA (1999), não há base concreta quanto às razões pelas quais a unidade é adicionada ao invés de outra constante, tais como 0,5 ou dois. Outros princípios alternativos foram propostos por HILL (1963), GRIFFITHS (1980) e BERRY (1987) para determinar o valor dessa constante $c$. Os procedimentos propostos por esses autores são aplicáveis em um determinado conjunto de pressupostos e requerem cálculos complexos. Devido a esse fato, um princípio prático foi proposto por YAMAMURA (1999), sugerindo como uma escolha razoável para o parâmetro $c$ o valor 0,5 , se a forma da distribuição não for conhecida. $\mathrm{O}$ autor defende o uso de $c$ igual a 0,5 porque uma distribuição definida em $(0$, $1,2, \ldots)$ pode ser aproximadamente descrita por uma distribuição contínua definida em $(0,8)$, admitindo-se $c=0,5$.

Este trabalho teve por objetivo encontrar uma transformação adequada para as variáveis observadas em experimentos com a cultura da abobrinha italiana, realizados em ambiente protegido, visando a estabilizar a variabilidade gerada pela presença de valores zeros nas múltiplas colheitas dos frutos.

\section{MATERIAL E MÉTODOS}

Os experimentos com abobrinha italiana, cultivar 'caserta', foram conduzidos em 2004, nas estações verão/outono (V/O) e inverno/primavera (I/ P), no Departamento de Fitotecnia da Universidade

Ciência Rural, v.39, n.6, set, 2009. 
Federal de Santa Maria, com latitude: $29^{\circ} 43$ 'S, longitude: $53^{\circ} 43$ ' $\mathrm{W}$ e altitude $95 \mathrm{~m}$, sendo o clima classificado como Cfa (subtropical úmido sem estação seca definida e com verões quentes) e solo classificado como Argissolo Vermelho Distrófico Arênico. Em cada estação, o cultivo foi realizado em um túnel plástico coberto com poliestireno de baixa densidade (PEDB) de 150 micras de espessura, com dimensões de $25 \mathrm{~m}$ de comprimento por $4 \mathrm{~m}$ de largura, com 3,5m de altura na parte central, utilizando irrigação por gotejamento, camalhões com $0,1 \mathrm{~m}$ de altura e $0,4 \mathrm{~m}$ de largura cobertos com mulching preto de PEDB de 35 micras, três linhas de cultivo, 25 plantas por linha, espaçamento entre plantas de $0,8 \mathrm{~m}$ e entre linhas de cultivo $1,2 \mathrm{~m}$. As aplicações de fungicidas e inseticidas e as desfolhas das plantas foram realizadas sempre que necessário nos dois túneis, conforme recomendação da cultura (FILGUEIRA, 2000).

Cada planta compôs uma parcela na linha de cultivo, sendo identificadas com o número de ordem da linha e da parcela dentro de cada linha. A definição do momento da colheita baseou-se no tamanho dos frutos, diariamente verificados e colhidos quando apresentaram comprimento acima de $18 \mathrm{~cm}$, colocados em sacos de papel e identificados, para posterior determinação, em cada colheita, do número de frutos colhidos por planta e da fitomassa fresca, utilizando balança digital com precisão de um grama. Em cada colheita realizada, as parcelas que não apresentaram frutos aptos a serem colhidos tiveram como valores das variáveis valor zero, indicando produção nula na colheita específica.

Utilizou-se a metodologia Box-Cox (BOX \& COX, 1964) para o conjunto de funções potências, para encontrar uma transformação para estabilizar ou reduzir a variabilidade existente entre os tratamentos simulados e normalizar os resíduos. Assim como para valores nulos, a família de transformações de Box-Cox fica restrita, avaliou-se a variável-resposta somada a uma constante. Adotou-se o procedimento proposto por YAMAMURA (1999), $\operatorname{com} c=0,5$, gerando as

expressões $f(y)=\frac{(y+0,5)^{\lambda}-1}{\lambda}, \lambda \neq 0$ e $f(y)=\ln (y+0,5)$,

$\lambda=0$, da família de transformações Box-Cox acrescida da constante $c=0,5$.

Para a escolha da melhor potência $\lambda$, foram considerados, para $\lambda$, valores no intervalo $[-2,2]$, conforme descrevem DRAPER \& SMITH (1998), sendo substituídos nas expressões $Z^{(\lambda)}=\frac{y^{\lambda}-1}{\lambda y^{\lambda-1}}, \lambda \neq 0$ ou $\mathrm{Z}^{(\lambda)}=y \ln y, \lambda=0$, em que o jacobiano $\mathrm{J}(\lambda, \mathrm{z})=1$, gerando valores transformados $\left(Z^{(\lambda)}\right)$ para cada variávelresposta. Para determinar o estimador de máxima verossimilhança $\left(\mathrm{L}_{\max }\right)$, substituiu-se a soma de quadrados residual $\left(\mathrm{SSE}_{(\lambda)}\right)$, encontrada para cada valor de $\lambda$, na expressão $L_{\max (\lambda)}=-\frac{n}{2} \ln \left(\frac{\hat{\sigma}_{\lambda}^{2}(z)}{n}\right)=-\frac{n}{2} \ln \left(\frac{\operatorname{SSE}(\lambda)}{n}\right)$, e o melhor $\lambda$ foi escolhido como aquele que proporcionou maior valor de $L_{\max }$.

Foi realizada a análise de variância (ANOVA) para as variáveis original e transformada. Para a realização da ANOVA, foi adotado o delineamento em blocos ao acaso em faixas, considerando como fator A a posição da planta dentro de linha de cultivo (25 plantas por linha) e como fator D as oito colheitas realizadas, simulando 200 tratamentos, com cada linha de cultivo como uma repetição. Antes a após a transformação dos dados foram aplicados testes de Lilliefors (CAMPOS, 1983), para verificar a aderência dos dados à distribuição normal e também testes de Bartlett para verificar a homocedasticidade dos erros. Posteriormente, foram obtidas as medidas descritivas e foi realizada a análise dos resíduos, antes e após a transformação. Para a análise dos dados, foram utilizados os softwares Statistica 7.0 e SAS, em nível de $5 \%$ de probabilidade de erro.

\section{RESULTADOS E DISCUSSÃO}

Observou-se que a frequência de valores zero, obtidos nas colheitas para o número e a fitomassa de frutos de abobrinha italiana, variou em torno de $40 \%$ no inverno/na primavera (I/P) e $60 \%$ no verão/ outono (V/O). Esse comportamento indica que a maior variabilidade para as variáveis estudadas foi obtida na estação de cultivo $\mathrm{V} / \mathrm{O}$, concordando com os resultados obtidos por SOUZA et al. (2002) e LÚCIO et al. (2008). A heterogeneidade das variâncias da fitomassa de frutos, entre plantas para as diferentes colheitas, devese, principalmente, à grande variabilidade existente entre o crescimento das plantas e dos frutos em condições de ambiente protegido, conforme descrevem SOUZA et al. (2002), MELLO et al. (2004), LORENTZ et al. (2005) e LÚCIO et al. (2008), causada pelas maiores variações nas condições ambientais nesse sistema de cultivo, tais como temperatura do ar, radiação solar global, nebulosidade e umidade relativa do ar, fatores mais proeminentes na estação $\mathrm{V} / \mathrm{O}$ que na estação I/P.

$O$ valor da potência $\lambda$ que minimiza a raiz do quadrado médio do erro (RQME) foi de 0,25 (Tabela 1), indicando que a melhor transformação para as variáveis-resposta foi a raiz quarta. A relação direta entre a fitomassa e o número de frutos colhidos pode 
Tabela 1 - Estimativas dos valores da função de máxima verossimilhança $\left(\mathrm{L}_{\max }\right)$ e da raiz do quadrado médio do erro (RQME) obtidas pelo procedimento Box-Cox na escolha da potência $\lambda$, para as variáveis número de frutos (NF), por planta e por colheita, e fitomassa fresca de frutos (FFF), em quilogramas por planta por colheita, de abobrinha italiana cultivada em ambiente protegido nas estações sazonais verão/outono (V/O) e inverno/primavera (I/P)). Santa Maria - RS. 2008.

\begin{tabular}{|c|c|c|c|c|c|c|c|c|}
\hline \multirow{3}{*}{$\lambda$} & \multicolumn{4}{|c|}{ 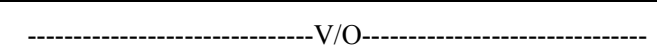 } & \multicolumn{4}{|c|}{ 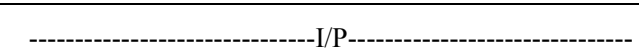 } \\
\hline & \multicolumn{2}{|c|}{------------NF------------' } & \multicolumn{2}{|c|}{-------------FFF------------ } & \multicolumn{2}{|c|}{ 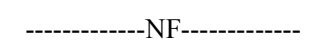 } & \multicolumn{2}{|c|}{------------FFF------------ } \\
\hline & $\mathrm{L}_{\max }$ & RQME & $\mathrm{L}_{\max }$ & RQME & $\mathrm{L}_{\max }$ & RQME & $\mathrm{L}_{\max }$ & RQME \\
\hline$-2,00$ & $-21147,13$ & 27,2278 & 19919,61 & 22,4867 & $-63204,66$ & 51,9505 & $-58751,00$ & 39,3280 \\
\hline$-1,75$ & $-18892,06$ & 9,1420 & $-17839,87$ & 16,2471 & $-56550,28$ & 34,2742 & $-52775,50$ & 27,0711 \\
\hline$-1,50$ & $-16746,51$ & 13,6897 & $-15874,04$ & 11,9497 & $-50204,73$ & 23,0530 & $-47127,26$ & 19,0193 \\
\hline$-1,25$ & $-14739,34$ & 10,0044 & $-14047,69$ & 8,9826 & $-44264,04$ & 15,9029 & $-41883,75$ & 13,7046 \\
\hline$-1,00$ & $-12908,27$ & 7,5151 & $-12392,71$ & 6,9356 & $-38859,58$ & 11,3444 & $-37143,21$ & 10,1904 \\
\hline$-0,75$ & $-11301,12$ & 5,8462 & $-10948,35$ & 5,5342 & $-34162,63$ & 8,4584 & $-33028,48$ & 7,8796 \\
\hline$-0,50$ & $-9974,77$ & 4,7520 & $-9761,29$ & 4,5972 & $-30374,99$ & 6,6755 & $-29687,65$ & 6,3948 \\
\hline$-0,25$ & $-8989,56$ & 4,0740 & $-8883,44$ & 4,0078 & $-27693,79$ & 5,6455 & $-27285,09$ & 5,5031 \\
\hline 0,00 & $-8397,61$ & 3,7141 & $-8365,66$ & 3,6963 & $-26252,50$ & 5,1592 & $-25974,24$ & 5,0702 \\
\hline 0,25 & $-8227,79$ & 3,6168 & $-8246,36$ & 3,6280 & $-26070,74$ & 5,1009 & $-25851,59$ & 5,0315 \\
\hline 0,50 & $-8475,82$ & 3,7597 & $-8538,66$ & 3,7976 & $-27053,03$ & 5,4239 & $-26914,73$ & 5,3772 \\
\hline 0,75 & $-9106,45$ & 4,1491 & $-9224,06$ & 4,2270 & $-29035,05$ & 6,1392 & $-29058,92$ & 6,1483 \\
\hline 1,00 & $-10066,08$ & 4,8202 & $-10257,88$ & 4,9681 & $-31838,01$ & 7,3146 & $-32117,44$ & 7,4435 \\
\hline 1,25 & $-11296,89$ & 5,8424 & $-11583,01$ & 6,1112 & $-35302,12$ & 9,0828 & $-35913,43$ & 9,4365 \\
\hline 1,50 & $-12746,33$ & 7,3273 & $-13142,91$ & 7,7983 & $-39297,64$ & 11,6593 & $-40292,85$ & 12,4075 \\
\hline 1,75 & $-14370,83$ & 9,4446 & $-14889,02$ & 10,2448 & $-43723,58$ & 15,3747 & $-45134,51$ & 16,7921 \\
\hline 2,00 & $-16135,99$ & 12,4441 & $-16782,76$ & 13,7731 & $-48502,26$ & 20,7260 & $-50347,16$ & 23,2591 \\
\hline
\end{tabular}

ter sido um dos motivos pelos quais se tem o mesmo valor $\lambda$ para essas duas variáveis. Já na tabela 2 encontram-se as medidas descritivas para o número e a fitomassa fresca de frutos com o acréscimo da constante, antes e após a transformação. Ao se aplicar a transformação na variável acrescida da constante, observou-se uma redução no coeficiente de variação, em comparação ao da variável original $+0,5$, indicando uma redução da variabilidade dos dados. Essa redução deve ser considerada interessante, pois o decréscimo no desvio padrão foi proporcionalmente maior que o aumento no valor da média, o que se espera de uma transformação de dados para homogeneizar variâncias.
Pelos resultados obtidos nas análises de variância para o número e a fitomassa fresca de frutos para as duas épocas de cultivo, verificou-se uma redução no quadrado médio do erro (QME) após o uso da transformação, de 90 e $96 \%$ na estação $\mathrm{V} / \mathrm{O}$, respectivamente, para número e fitomassa fresca de frutos, e de $92 \%$ na estação I/P, para as duas variáveis estudadas (Tabela 2). Esse resultado é importante, pois quanto maior o erro, menor a probabilidade de serem encontradas diferenças significativas entre médias de tratamentos. Portanto, a transformação raiz quarta $\left(y^{0,25}\right)$ para o número e o peso de frutos de abobrinha italiana permitiu uma redução da variabilidade.

Tabela 2 - Média ( $\overline{\mathrm{X}})$, desvio padrão (s), coeficiente de variação (CV\%) e quadrado médio do erro (QMe) das variáveis originais e transformadas mais constante para número de frutos (NF), por planta por colheita, e fitomassa fresca de frutos (FFF), em quilogramas por planta por colheita, em experimentos com abobrinha italiana cultivada em ambiente protegido nas estações sazonais verão/outono (V/O) e inverno/primavera (I/P). Santa Maria - RS. 2008.

\begin{tabular}{|c|c|c|c|c|c|c|c|c|}
\hline \multirow{2}{*}{ Variáveis } & \multicolumn{4}{|c|}{ 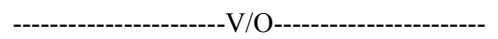 } & \multicolumn{4}{|c|}{ 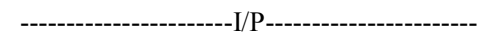 } \\
\hline & $\bar{X}$ & $\mathrm{~s}$ & $\mathrm{CV}(\%)$ & QMe & $\overline{\mathrm{X}}$ & $\mathrm{s}$ & $\mathrm{CV}(\%)$ & QMe \\
\hline $\mathrm{FFF}+0,5$ & 0,09 & 0,15 & 166,67 & 0,53 & 0,19 & 0,23 & 121,05 & 0,04 \\
\hline Transformação (FFF+0,5) & 0,87 & 0,05 & 5,75 & 0,02 & 0,91 & 0,07 & 7,69 & 0,003 \\
\hline $\mathrm{NF}+0,5$ & 0,53 & 0,79 & 149,06 & 0,02 & 0,81 & 0,82 & 101,23 & 0,51 \\
\hline Transformação (NF + 0,5) & 0,96 & 0,16 & 16,67 & 0,002 & 1,03 & 0,17 & 16,50 & 0,04 \\
\hline
\end{tabular}

Ciência Rural, v.39, n.6, set, 2009. 
Com a aplicação do teste de Lilliefors, observou-se que a aderência dos dados à distribuição normal foi atendida, tanto com os dados originais, quanto após a transformação via raiz quarta. Esse resultado, além de viabilizar a utilização do teste de Bartlett, indica que, apesar do excesso de zeros no banco de dados e da presença de superdispersão, as variáveis estudadas apresentaram melhoras significativas em relação ao atendimento à normalidade dos erros, verificado na figura 1 , com os dados transformados para ambas as variáveis.

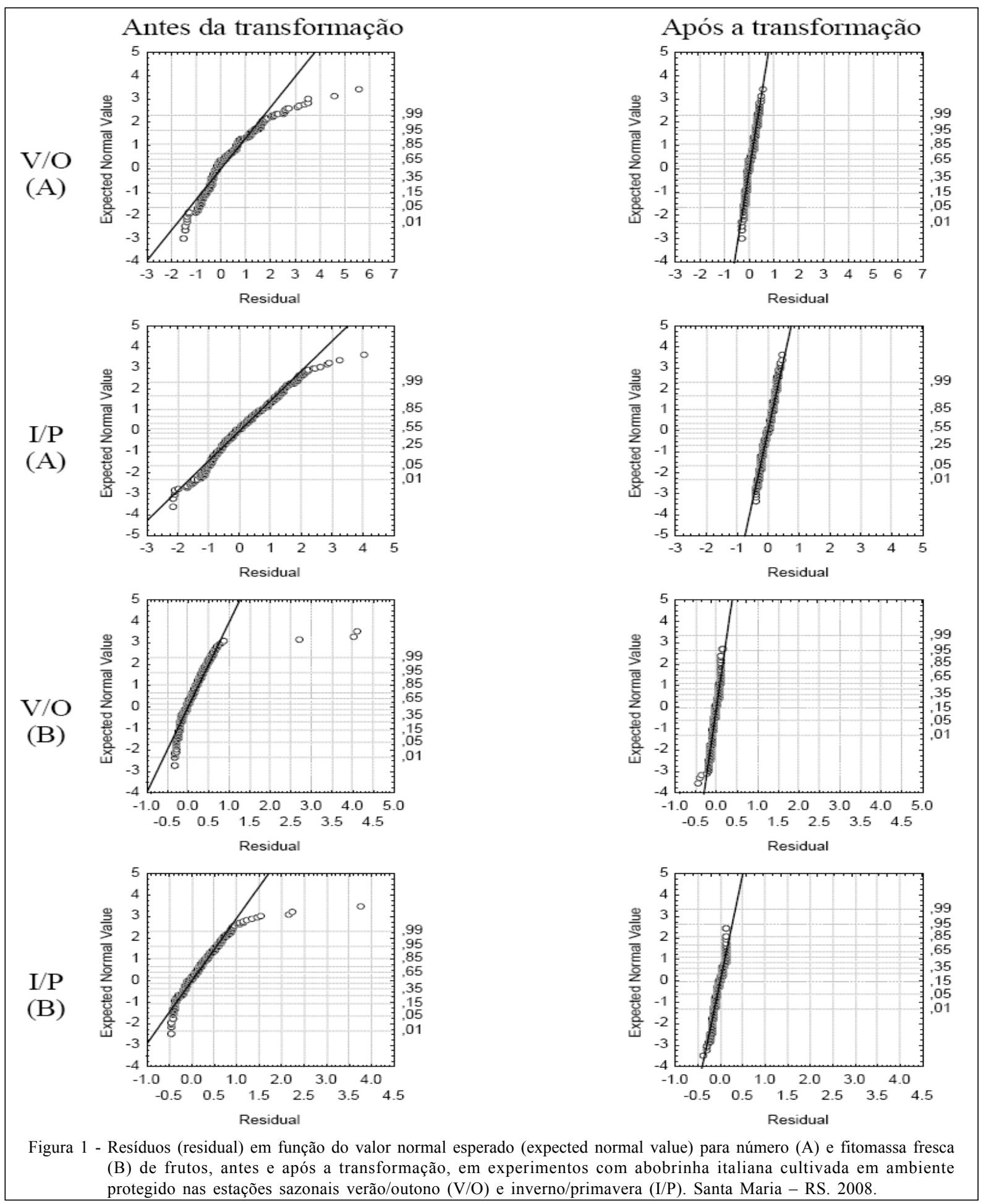

Ciência Rural, v.39, n.6, set, 2009. 
A verificação da homogeneidade de variâncias se fez necessária para observar se o emprego da transformação raiz quarta $\left(y^{0,25}\right)$ foi eficiente para adequar os dados à pressuposição da homogeneidade das variâncias residuais. Os resultados do teste de Bartlett mostraram que a transformação raiz quarta $\left(y^{0,25}\right)$ não foi eficiente para adequar os dados a essa pressuposição, pois foi rejeitada a hipótese de homocedasticidade para o número e a fitomassa fresca de frutos nas duas épocas, apesar da redução no valor do $\chi^{2}$ calculado para as duas variáveis avaliadas, após a aplicação da transformação. Sabe-se que muitas circunstâncias podem interferir na violação da pressuposição de homogeneidade de variância. Em geral, a heterogeneidade da variância pode estar associada com variáveis-resposta cuja distribuição não é normal e cuja variância relaciona-se com a média em decorrência da própria forma da distribuição.

Mesmo se rejeitando a hipótese de homocedasticidade das variâncias residuais, o nível de significância apresentado no teste, após a transformação, foi próximo do adotado no estudo $(\mathrm{P}<$ $0,05)$, além da transformação raiz quarta apresentar a menor relação entre RQME e $\mathrm{L}_{\max }$. Assim, essa transformação é apresentada como uma alternativa para estabilizar a variância ou, pelo menos, reduzir a variabilidade existente, auxiliando nas análises estatísticas e interpretações dos resultados desse tipo de experimento, sem comprometer a precisão experimental.

Usualmente, em experimentos com olericolas realizados em ambiente protegido, as parcelas experimentais são definidas com uma única planta, devido à limitação de área, favorecendo assim o surgimento de valores zero para as variáveis que indicam a produção de frutos no momento da colheita. Essa condição gera a superdispersão e os problemas subsequentes nas análises estatísticas dos dados que podem ser contornados com a aplicação de transformação de dados ou com a abordagem via modelos lineares generalizados. Essas alternativas podem ser aplicadas após a realização do experimento, o que nem sempre está à disposição do pesquisador responsável pelo trabalho. Dessa forma, o planejamento de experimentos com parcelas compostas de mais de uma planta, combinados com diferentes agrupamentos de colheitas, pode ser uma solução mais prática para evitar ou minimizar a presença de valores zero no banco de dados, reduzindo assim a superdispersão e seus efeitos nas análises estatísticas.

\section{CONCLUSÃO}

Para o número e a fitomassa fresca de frutos da abobrinha italiana, a transformação indicada é a raiz quarta.

\section{AGRADECIMENTOS}

Ao Conselho Nacional de Desenvolvimento Científico e Tecnológico (CNPq), pelo auxílio financeiro para a realização do trabalho e pela concessão de bolsas de produtividade em pesquisa aos autores Alessandro Dal'Col Lúcio e Sidinei José Lopes.

\section{REFERÊNCIAS}

BANZATTO, D.A.; KRONKA, S.N. Experimentação agrícola. 4.ed. Jaboticabal: FUNEP, 2006. 246p.

BERRY, D.A. Logarithmic transformations in ANOVA. Biometrics, Washington, v.43, p.439-456, 1987.

BORGATTO, A.F. et al. Modelos para proporções com superdispersão e excesso de zeros - Um procedimento Bayesiano. Revista de Matemática e Estatística, São Paulo, v.24, n.2, p.121-131, 2006.

BOX, G.E.P.; COX, D.R. An analysis of transformations. Journal of the Royal Statistical Society. Series B (Statistical Methodological), New York. v.26, n.2, p.211$252,1964$.

CAMARGO. L.S. As hortaliças e seu cultivo. Campinas: Fundação Cargill, 1981. 321p.

CAMPOS, H. Estatística experimental não-paramétrica. 4.ed. Piracicaba: ESALQ, 1983. 349p.

CONAGIN, A. et al. Efeito da falta de normalidade em testes de homogeneidade das variâncias. Bragantia, Campinas, v.52, n.2, p.173-180, 1993. Disponível em: <http://www.scielo.br/ scielo.php?script=sci_art text \& pid $=$ S $0006-$ $87051993000200010 \& \operatorname{lng}=$ en $\&$ nrm $=$ iso\&tlng $=p t>$ Acesso em: 11 de novembro de 2008. Doi: 10.1590/S000687051993000200010 .

DRAPER, N.R.; SMITH, H. Applied regression analysis. 3.ed. New York: John Wiley, 1998. 706p.

FILGUEIRA, F.A.R. Novo manual de olericultura: tecnologia moderna na produção e comercialização de hortaliças. Viçosa: UFV, 2000. 402p.

GRIFFITHS, D.A. Interval estimation for the three-parameter lognormal distribution via the likelihood function. Journal of the Royal Statistical Society. Series C (Applied Statistics), New York, v.29, n.1, p.58-68, 1980.

HILL, B.M. The three-parameter lognormal distribuition and Bayesian analysis of a point-source epidemic. Journal of the American Statistical Association, Alexandria. v.58, n.301, p.72-84, 1963 .

Ciência Rural, v.39, n.6, set, 2009. 
LORENTZ, L.H. et al. Variabilidade da produção de frutos de pimentão em estufa plástica. Ciência Rural, Santa Maria, v.35, n.2, p.316-323, 2005. Disponível em: <http:// www.scielo.br/scielo.php?script $=$ sci arttext\&pid $=\mathrm{S} 0103$ $84782005000200011 \& \operatorname{lng}=\mathrm{en} \& \mathrm{nrm}=\mathrm{iso} \& \mathrm{t} \operatorname{lng}=\mathrm{pt}>$. Acesso em 28 de maio de 2008. Doi: 10.1590/S010384782005000200011

LOPES, S.J. et al. Técnicas experimentais para tomateiro tipo salada sob estufas plásticas. Ciência Rural, Santa Maria v.28, n.2, p.193-197, 1998. Disponível em: <http:// w w w.s c i e 1 o.br/s c i e 1 o.p h p ? p id = S 0103 $84781998000200002 \& \mathrm{script}=\mathrm{sci}$ arttext $\& \operatorname{tn} \mathrm{ln}=\mathrm{e}>$. Acesso em: 21 de maio de 2008. Doi: 10.1590/S010384781998000200002

LÚCIO, A.D. et al. Tamanho da amostra e método de amostragem para avaliação de características do pimentão em estufa plástica. Horticultura Brasileira, Brasília, v.21, n.2, p.181-185, 2003. Disponível em $<$ http://www.scielo.br/scielo.php?pid=S0102$05362003000200012 \&$ script $=$ sci_arttext $\&$ tlng $=\mathrm{pt}>$. Acesso em 15 de agosto de 2008. Doi: 10.1590/S010205362003000200012 .

LÚCIO, A.D. et al. Variância e média da massa de frutos de abobrinha-italiana em múltiplas colheitas. Horticultura Brasileira, Brasília, v.26, n.3, p.335-341, 2008. Disponível em: $<$ http://www.scielo.br/scielo.php?pid=S 0102 $05362008000300009 \&$ script $=$ sci_arttext $\&$ tlng $=\mathrm{g}>$. Acesso em
23 de janeiro de 2009. Doi: 10.1590/S010205362008000300009 .

MELLO, R.M. et al. Size and form of plots for the culture of the italian pumpkin in plastic greenhouse. Scientia Agricola, Piracicaba, v.61, n.4, p.457-461, 2004. Disponível em < http:/ / w w w. s c i e 1 o.b r / s c i e 1 o.p h p ? p i d = S 0103 $90162004000400017 \&$ script $=$ sci_arttext $\&$ tlng $=$ en $>$ Acesso em: 05 de maio de 2008. Doi: 10.1590/S010390162004000400017.1

SOUZA, M.F. et al. Tamanho da amostra para peso da massa de frutos, na cultura da abóbora italiana em estufa plástica. Revista Brasileira de Agrociência, Pelotas, v.8, n.2, p.123-128, 2002.

STORCK, L. et al. Experimentação vegetal. Santa Maria: UFSM, 2000. 198p.

STRECK, L. et al. Evapotranspiração máxima e coeficiente de cultura da abóbora italiana em estufa plástica. Revista Brasileira de Agrometeorologia, Santa Maria, v.11, n.1, p.43-52, 2003.

YAMAMURA, K. Transformation using $(\mathrm{x}+0.5)$ to stabilize the variance of populations. Journal Researches on Population Ecology, Tokyo, v.41, n.3, p.229-234, 1999. Disponível em <http://www.springerlink.com/content/ ryhwj0c7c79qq8t7/fulltext.pdf $>$. Acesso em 10 de junho de 2008. Doi: $10.1007 / \mathrm{s} 101440050026$. 